\title{
Oxylipin signaling: a distinct role for the jasmonic acid precursor cis-(+)-12-oxo-phytodienoic acid (cis-OPDA)
}

\author{
Anuja Dave and lan A. Graham* \\ Department of Biology, Centre for Novel Agricultural Products, University of York, York, UK
}

Edited by:

Kent D. Chapman, University of North

Texas, USA

\section{Reviewed by:}

Clay Carter, University of Minnesota

Duluth, USA

Ivo Feussner,

Georg-August-University Goettingen, Germany

\section{${ }^{*}$ Correspondence:}

Ian A. Graham, Department of

Biology, Centre for Novel Agricultural

Products, University of York,

Heslington, York YO10 5DD, UK.

e-mail: ian.graham@york.ac.uk
Oxylipins are lipid-derived compounds, many of which act as signals in the plant response to biotic and abiotic stress. They include the phytohormone jasmonic acid (JA) and related jasmonate metabolites cis-(+)-12-oxo-phytodienoic acid (cis-OPDA), methyl jasmonate, and jasmonoyl-L-isoleucine (JA-lle). Besides the defense response, jasmonates are involved in plant growth and development and regulate a range of processes including glandular trichome development, reproduction, root growth, and senescence. cis-OPDA is known to possess a signaling role distinct from JA-lle. The non-enzymatically derived phytoprostanes are structurally similar to cis-OPDA and induce a common set of genes that are not responsive to JA in Arabidopsis thaliana. A novel role for cis-OPDA in seed germination regulation has recently been uncovered based on evidence from double mutants and feeding experiments showing that cis-OPDA interacts with abscisic acid (ABA), inhibits seed germination, and increases ABA INSENSITIVE5 (ABI5) protein abundance. Large amounts of cis-OPDA are esterified to galactolipids in $A$. thaliana and the resulting compounds, known as Arabidopsides, are thought to act as a rapidly available source of cis-OPDA.

Keywords: oxylipins, jasmonates, 12-oxo-phytodienoic acid, jasmonic acid, phytoprostanes, seed germination, seed dormancy, lipid signaling

\section{SYNTHESIS OF OXYLIPINS}

Oxylipins are a diverse group of lipid-derived signaling compounds that are generated following oxidation of polyunsaturated fatty acids (PUFAs) such as linoleic acid (18:2), octadecatrienoic acid (18:3n-3), and hexadecatrienoic acid (16:3n-3; Wasternack, 2007; Mosblech et al., 2009; Wasternack and Kombrink, 2010). These fatty acids are released from plastidial membrane lipids by lipases including DEFECTIVE IN ANTHER DEHISCENCE1 (DAD1) and DONGLE (DGL; Ishiguro et al., 2001; Hyun et al., 2008; Ellinger et al., 2010) and are subsequently oxidized by lipoxygenases (LOX) to form hydroperoxides (Vick and Zimmerman, 1983; Bell et al., 1995). As shown in Figure 1, the octadecanoid pathway in Arabidopsis thaliana that gives rise to jasmonic acid (JA), initiates in the plastid with the oxidation of octadecatrienoic acid (18:3n-3) by 13-lipoxygenase (13-LOX) to form 13 -hydroperoxylinolenic acid. This is then acted on by allene oxide synthase (AOS) and allene oxide cyclase (AOC) to give cis-(+)-12oxo-phytodienoic acid (cis-OPDA). cis-OPDA then travels via the cytosol to the peroxisome with uptake into this organelle being mediated, at least in part, by the ATP binding cassette (ABC) transporter protein, COMATOSE (CTS; Theodoulou et al., 2005). Once in the peroxisome cis-OPDA is reduced (Sanders et al., 2000; Schaller et al., 2000; Stintzi and Browse, 2000) and activated to the CoA ester (Schneider et al., 2005; Koo et al., 2006; Kienow et al., 2008) prior to undergoing three rounds of $\beta$-oxidation to form JA (Cruz Castillo et al., 2004; Pinfield-Wells et al., 2005; Schilmiller et al., 2007; Figure 1).

In addition to the action of AOS on plastidial fatty acid hydroperoxides, they are also cleaved by hydroperoxide lyases (HPLs) to produce $\mathrm{C}_{6}$-aldehydes such as $(2 E)$-hexenal,
(3Z)-hexenal and their volatile derivatives termed collectively as green leaf volatiles (GLVs; Chehab et al., 2008). HPL and AOS compete for hydroperoxide substrates and it has been shown that JA and cis-OPDA accumulation are reduced upon HPL overexpression (Chehab et al., 2008). These authors also showed that JA is involved in the direct defense response while the GLV hexenyl acetate mediates the indirect defense response. Moreover, HPL activity is not indispensable for normal growth, development, or defense since it has been shown that functional HPL activity is absent in the A. thaliana ecotype Col-0 (Duan et al., 2005).

A third branch of the oxylipin biosynthetic pathway originating from plastidial hydroperoxides originates through the activity of divinyl ether synthases that produce divinyl ether oxylipins which have also been shown to play a role in plant defense in a number of systems (Weber et al., 1999; Itoh and Howe, 2001). However, Eschen-Lippold et al. (2010) have more recently reported that oxylipins resulting from the action of divinyl ether synthase are not required for the $R$-gene-mediated resistance in potato.

While much is known about the physiological role of JA and the mechanistic basis of how jasmonate signaling operates (Browse, 2009), much less is understood about the biological functions of the other oxylipins. This mini-review will summarize recent developments in our understanding of the role played by the JA precursor cis-OPDA and the structurally similar phytoprostanes, which are synthesized by a non-enzymatic route.

\section{A DISTINCT ROLE FOR cis-OPDA IN PLANT SIGNALING}

cis-OPDA, JA, JA-Ile, and methyl jasmonate (MeJA) are collectively referred to as jasmonates which in addition to the involvement in the response to stress also play a role in regulating processes such as 


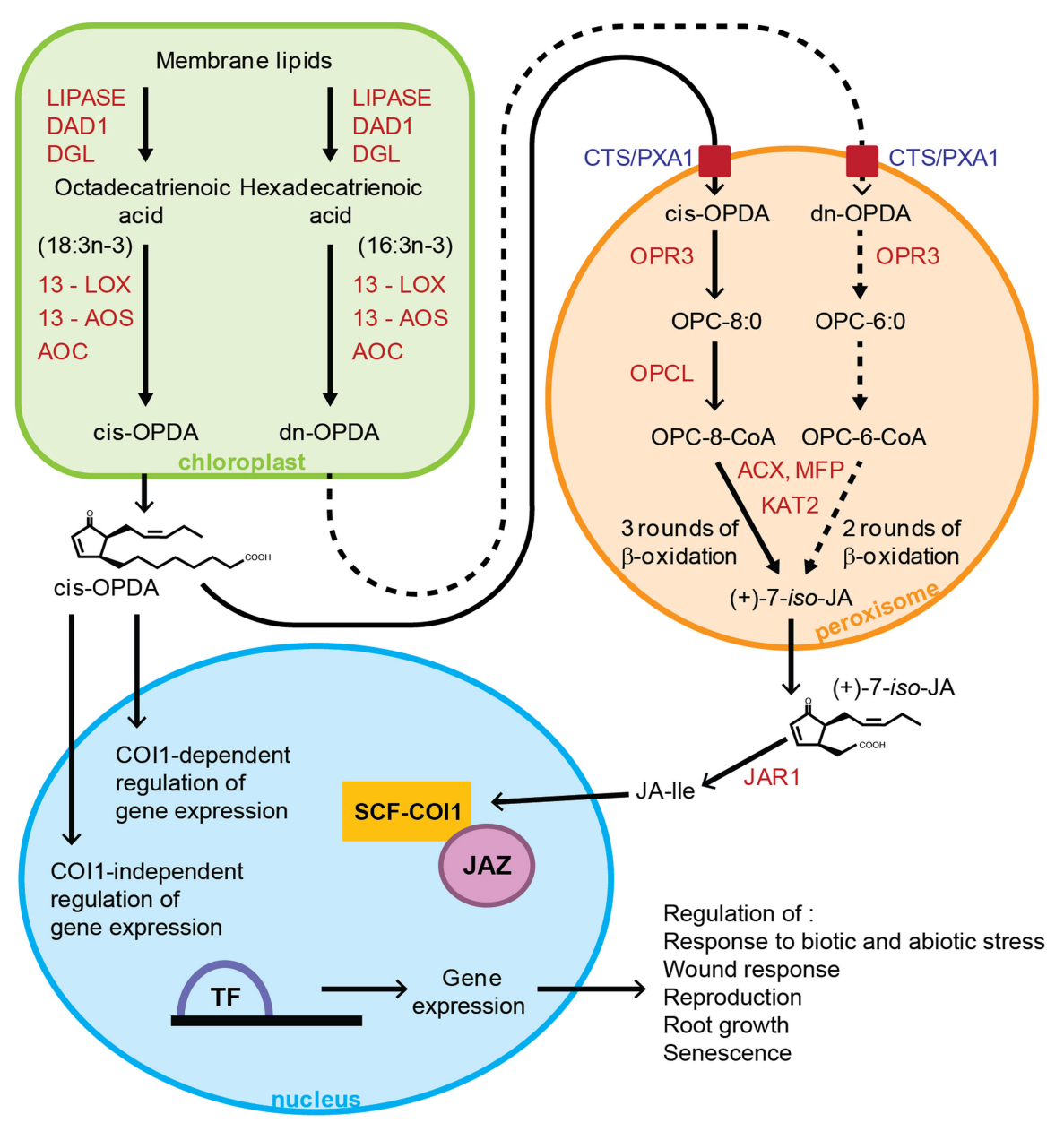

FIGURE 1 | Oxylipin biosynthesis pathway and signal transduction in Arabidopsis thaliana. JA biosynthesis initiates in the plastid with release of octadecatrienoic acid or hexadecatrienoic acid from membrane lipids by lipases such as DAD1 and DGL. cis-OPDA and dn-OPDA are formed following sequential steps catalyzed by 13-LOX, 13-AOS, and AOC. cis-OPDA is transported to the peroxisome via the CTS transporter, where after reduction by OPR3, OPC-8:0 is formed. This is activated to its CoA ester by OPCL1, which then undergoes three rounds of $\beta$-oxidation catalyzed by ACX, KAT, and MFP to give (+)-7-iso-JA. JAR1 catalyzes formation of the amino acid conjugate JA-Ile from JA in the cytosol, which is the active form of the hormone involved in JA signaling. JAZ proteins repress expression of JA-responsive genes. In response to JA-lle, the JAZ proteins are targeted by SCF ${ }^{\mathrm{CO} I 1}$ for degradation, thus leading to JA-dependent gene expression and ultimately the regulation of various physiological processes. The model proposes formation of the COI1-JAZ complex in the nucleus. cis-OPDA's regulation of gene expression can be COI1-dependent or COI1 independent, although cis-OPDA has not been shown to promote binding of COI1 and JAZ. Enzyme names are shown in red. Dashed arrows indicate route to JA biosynthesis via dn-OPDA, where these steps are yet to be proven experimentally. DAD1, DEFECTIVE IN ANTHER DEHISCENCE1; DGL, DONGLE; 13-LOX, 13-lipoxygenase; 13-AOS, 13-allene oxide synthase; AOC, allene oxide cyclase; OPR3, 12-oxophytodienoate reductase3; OPCL1, OPC-8:CoA ligase1; CTS, COMATOSE; ACX, acyl CoA oxidase; KAT, 3-I-ketoacyl-CoA-thiolase; MFP, multifunctional protein; JA, jasmonic acid; cis-OPDA,

cis-(+)-12-oxo-phytodienoic acid; dn-OPDA, dinor-oxo-phytodienoic acid; JA-Ile, jasmonoyl-L-isoleucine; COI1, CORONATINE-INSENSITIVE1; JAZ, jasmonate ZIM domain. root growth, tendril coiling, senescence, glandular trichome development, and reproduction (Staswick et al., 1992; Feys et al., 1994; Xie et al., 1998; Li et al., 2004; Balbi and Devoto, 2008; Wasternack and Kombrink, 2010). By far the best characterized jasmonate signaling mechanism is the transcriptional control of JA-responsive genes. JA is conjugated to amino acids in A. thaliana by an enzyme encoded by JAR1 (Staswick and Tiryaki, 2004). One such conjugate, jasmonoyl-L-isoleucine (JA-Ile), rather than JA or cis-OPDA plays the crucial role in transcriptional control via the jasmonate ZIM domain (JAZ) repressor proteins (Chini et al., 2007; Thines et al., 2007; Yan et al., 2007). JA-Ile promotes binding of the
F-box protein CORONATINE-INSENSITIVE1 (COI1) and JAZ proteins resulting in the degradation of JAZ proteins by the 26Sproteasome (Chini et al., 2007; Thines et al., 2007). A cytochrome P450 encoded by CYP94B3 metabolizes JA-Ile to 12OH-JA-Ile (Kitaoka et al., 2011; Koo et al., 2011; Heitz et al., 2012) which is less effective than JA-Ile at promoting COI1-JAZ binding (Koo et al., 2011), thus suggesting a role for this enzyme in the inactivation of JA-Ile and attenuation of the jasmonate response (Koo et al., 2011). An additional enzyme, CYP94C1 is also involved in the oxidative catabolism of JA-Ile, by converting it to $12 \mathrm{COOH}-\mathrm{JA}-\mathrm{Ile}$ (Heitz et al., 2012). 
That signals other than JA-Ile are involved in oxylipin signaling was suggested through the use of a mutant defective in 12-oxophytodienoate reductase3 (opr3), which is compromised in the conversion of cis-OPDA to JA (Stintzi et al., 2001), yet it still undergoes a defense response. Treating the opr3 mutant with cisOPDA revealed two separate downstream signaling pathways, one dependent on COI1 and the other independent (Stintzi et al., 2001). In the case of the A. thaliana defense response, JA, and cis-OPDA appear to act in concert to fine tune the expression of defense genes. However, in other cases the roles of JA and cisOPDA are distinct as demonstrated for example by the fact that the male sterility phenotype of opr3 is rescued by JA but not cisOPDA (Stintzi and Browse, 2000). A recent publication reports that opr3 is not a complete null mutant and concludes that the defense response displayed by opr3 plants against a necrotrophic fungus is likely due to JA and not cis-OPDA (Chehab et al., 2011). Nevertheless, data from other publications do strongly suggest that cis-OPDA is capable of distinct signaling (Weiler et al., 1993; Blechert et al., 1999; Taki et al., 2005; Mueller et al., 2008; Ribot et al., 2008; Böttcher and Pollmann, 2009; Dave et al., 2011; Schäfer et al., 2011). In the tendril coiling response of Bryonia dioica, (Weiler et al., 1993; Blechert et al., 1999), the cis-OPDA-methyl ester acts faster and requires a much lower concentration than MeJA to elicit the response (Weiler et al., 1993). Taki et al. (2005) showed that in addition to a set of genes whose expression is induced by both JA and cis-OPDA, a subset of 157 of the 21,500 genes analyzed were found to be induced by cis-OPDA but not JA or MeJA. Half of these cis-OPDA-specific response genes (ORGs) were induced by wounding but their regulation was found to be COI1 independent (Taki et al., 2005). Similarly, the PHO1;H10 gene in $A$. thaliana which is induced by various abiotic and biotic stresses, responds to cis-OPDA application, but not JA and in this case the effect of cis-OPDA is COI1-dependent (Ribot et al., 2008).

While a number of signaling roles have been demonstrated for cis-OPDA, the 16 carbon homolog dinor-oxo-phytodienoic acid (dn-OPDA) which is synthesized from hexadecatrienoic acid via a parallel hexadecanoid pathway (Weber et al., 1997; Acosta and Farmer, 2010; Figure 1) has not as yet had any signaling function ascribed.

\section{cis-OPDA AS A NEW PLAYER IN SEED GERMINATION CONTROL}

We recently uncovered an additional role for cis-OPDA when investigating the mechanism by which the $\mathrm{ABC}$ transporter COMATOSE (CTS) regulates germination potential in A. thaliana (Dave et al., 2011). The severely impaired germination phenotype of cts mutants is also observed in other mutants that are compromised in peroxisomal $\beta$-oxidation, including kat2, acx 1 acx2, and csy2 csy3 (Pinfield-Wells et al., 2005; Pracharoenwattana et al., 2005; Footitt et al., 2006). Since JA synthesis is dependent on the uptake of cis-OPDA into peroxisomes (Acosta and Farmer, 2010 ) and three rounds of peroxisomal $\beta$-oxidation (Cruz Castillo et al., 2004; Pinfield-Wells et al., 2005; Schilmiller et al., 2007) we analyzed oxylipin levels in mutant seed to establish if there is any correlation with germination potential. Surprisingly, we found elevated levels of not only cis-OPDA but also JA and JA-Ile in the cts and $\beta$-oxidation mutants compared to wild type. Previously, we had quantified JA in wounded and unwounded leaves of the cts mutant and found that although levels were reduced relative to wild type they were still quantifiable, suggesting that while the CTS transporter is involved in peroxisome import, another transport mechanism such as ion trapping may also operate (Theodoulou et al., 2005). Analysis of developing seeds revealed that the oxylipins accumulate during late seed maturation and double mutant analysis revealed that cis-OPDA rather than JA or JA-Ile contributes to the block in seed germination in A. thaliana (Dave et al., 2011). Seed treatments revealed that cis-OPDA is much more effective than JA at inhibiting wild type seed germination and this effect is independent of COI1 but synergistic with the seed germination antagonist, abscisic acid (ABA). The ABA INSENSITIVE5 (ABI5) locus rescues the impaired germination phenotype of ped3, an allele of cts (Kanai et al., 2010). Consistent with these observations we found that cis-OPDA treatment increased ABI5 protein abundance in a manner that parallels the inhibitory effect of cis-OPDA and cis-OPDA + ABA on seed germination. Previous results from our laboratory showed that ABI5 is expressed specifically in the micropylar region of the single cell endosperm layer through which the radicle has to emerge for germination to proceed in A. thaliana (Penfield et al., 2006). The work of Kanai et al. (2010) highlights the correlation between ABI5 transcripts and those encoding polygalacturonase inhibiting proteins (PGIPs) which reduce cell wall pectin degradation. Thus we can propose a mechanism by which cis-OPDA together with ABA controls protein levels of the ABI5 transcription factor and this in turn regulates abundance of the PGIPs at the micropylar region of the endosperm, and in so doing determines whether or not the radicle can break through the endosperm barrier leading to seed germination. Our work is now focused on elucidating the mechanism by which cis-OPDA operates to control levels of proteins such as ABI5 and what regulates cis-OPDA levels in developing wild type seeds.

\section{CHEMICALLY REACTIVE CYCLOPENTENONE OXYLIPINS}

Various stress stimuli, such as wounding and pathogen infection, result in the activation of biosynthetic enzymes responsible for accumulation of cis-OPDA and JA (Wasternack, 2007; Mosblech et al., 2009). In addition to this enzymatic route, a non-enzymatic route for oxylipin formation triggered by reactive oxygen species (ROS) and free radicals also operates to produce an array of oxidized lipids including phytoprostanes and hydroxy fatty acids (Imbusch and Mueller, 2000; Mosblech et al., 2009). Phytoprostanes and cis-OPDA are structurally similar cyclopentenones that contain a chemically reactive $\alpha, \beta$-unsaturated carbonyl structure that can bind to free thiol groups and modify cellular proteins. This has led to their classification as reactive electrophilic species (RES) and it has been proposed that this RES subgroup of oxylipins induce a common cluster of defense genes (Almeras et al., 2003; Weber et al., 2004; Farmer and Davoine, 2007) but other reports indicate that chemical reactivity and gene expression do not always correlate (Mueller et al., 2008). Microarray analysis in A. thaliana has shown that phytoprostanes and cis-OPDA induce the expression of genes associated with cellular detoxification, stress responses, and secondary metabolism with 60 and $30 \%$ of the genes induced by phytoprostanes and cis-OPDA respectively being dependent on the basic leucine zipper containing TGA class of 
transcription factors in A. thaliana (Mueller et al., 2008; Mosblech et al., 2009). JA, which is a cyclopentanone and much less chemically reactive, does not induce this same group of genes. Furthermore, there was no significant overlap observed between the cyclopentenone oxylipin regulated genes described by Mueller et al. (2008) and genes altered in expression in cts developing seeds that have high levels of both JA and cis-OPDA (Dave et al., 2011). Much remains to be done to establish the details of how these various oxylipins are perceived and how the resulting signals elicit the variety of observed responses.

\section{ARABIDOPSIDES}

Galactolipids containing esterified cis-OPDA and dn-OPDA have been found in A. thaliana and some other related species of the genus Arabidopsis, and these complex lipids are referred to as Arabidopsides (Stelmach et al., 2001; Hisamatsu et al., 2003, 2005; Andersson et al., 2006; Buseman et al., 2006; Böttcher and Weiler, 2007; Kourtchenko et al., 2007). A number of Arabidopsides have been identified and named according to the position at which cis-OPDA is found esterified to the monogalactosyl diacylglycerol (MGDG) or digalactosyl diacylglycerol (DGDG) instead of the fatty acyl moiety. For example Arabidopside A and Arabidopside $C$ are MGDG and DGDG derivatives respectively containing $c i s$-OPDA esterified at positions $s n-1$ and dn-OPDA at $s n-2$ positions (Hisamatsu et al., 2003, 2005). Arabidopsides are reported to accumulate following wounding of leaves (Buseman et al., 2006; Böttcher and Weiler, 2007; Kourtchenko et al., 2007). Kourtchenko et al. (2007) also demonstrated that Arabidopsides accumulate during the hypersensitive response to bacterial pathogens. Moreover, they show that in both the wounding and hypersensitive responses, Arabidopside formation is dependent on intact JA signaling as levels of Arabidopsides are severely reduced in the coil and jar 1 mutants compared to wild type following the two stimuli. Recently Vu et al. (2012) have reported that the basal composition of these complex oxidized lipids is different from those that are formed following various stress treatments. Based on the rapid and large increase in Arabidopsides following wounding, Buseman et al. (2006) hypothesize that galactolipids are the substrates of cis-OPDA/dn-OPDA synthesizing enzymes rather than free fatty acids being converted to cis-OPDA/dn-OPDA and then esterified to the galactolipids. This suggests that enzymes involved in cis-OPDA/dn-OPDA biosynthesis can act not only on free fatty acids, but also on lipid-bound fatty acids.

\section{REFERENCES}

Acosta, I. F., and Farmer, E. E. (2010). Jasmonates: The Arabidopsis Book. Rockville, MD: American Society of Plant Biologists.

Almeras, E., Stolz, S., Vollenweider, S., Reymond, P., MeneSaffrane, L., and Farmer, E. E. (2003). Reactive electrophile species activate defense gene expression in Arabidopsis. Plant J. 34, 205-216.

Andersson, M. X., Hamberg, M., Kourtchenko, O., Brunnström, A., McPhail, K.

A number of functions have been described for Arabidopsides. It has been hypothesized that they may function as a storage pool that could release cis-OPDA for direct signaling or as a substrate for production of JA (Kourtchenko et al., 2007; Ibrahim et al., 2011). Stelmach et al. (2001) have shown that $c i s-O P D A$ at the $s n-1$ position in Arabidopsides can be released by $s n$-1-specific lipases from Rhizopus arrhizus. Schäfer et al. (2011) report that lipase activity of grasshopper oral secretions are instrumental in release of cis-OPDA from Arabidopsides and hence play a role in defense response to herbivory. Some of the Arabidopsides display growthinhibiting effects on bacterial and fungal pathogens (Andersson et al., 2006; Kourtchenko et al., 2007). A senescence promoting effect for Arabidopside A has also been described (Hisamatsu et al., 2006).

As Arabidopsides have so far only been detected from a very limited range of species from the genus Arabidopsis it would appear that either these compounds are present in miniscule amounts in other plants or are completely absent (Mosblech et al., 2009). Based on current evidence it appears that this intriguing class of complex lipids do not have a generic role across species but have arisen by adaptation in just a few (Böttcher and Weiler, 2007).

\section{CONCLUSION}

A wide array of oxylipins are generated in response to various environmental stimuli and developmental cues. In some cases, such as plant defense, multiple oxylipins are involved while in others, such as reproductive development and seed germination, JA, and cis-OPDA respectively play the main role. Fine-tuning of jasmonate levels by factors such as environmental stress and developmental stage of the tissue is obviously important in eliciting a physiological response. Equally important is the responsiveness of different cell and tissue types to specific oxylipins or combinations of different oxylipins. Our recent demonstration of a specific role for $c i s$-OPDA in regulating germination potential in developing seeds provides an opportunity to further dissect the underlying mechanism. Establishing the role played by oxylipin signaling in the environmental and genetic control of seed dormancy and germination is an important challenge for the future.

\section{ACKNOWLEDGMENTS}

We acknowledge financial support from the UK Biotechnology and Biological Sciences Research Council (BBSRC) grant number BB/J00216X/1 for work on cis-OPDA.

Bell, E., Creelman, R. A., and Mullet, J. E. (1995). A chloroplast lipoxygenase is required for wound-induced jasmonic acid accumulation in Arabidopsis. Proc. Natl. Acad. Sci. U.S.A. 92, 8675-8679.

Blechert, S., Bockelmann, C., Füßlein, M., Schrader, T. V., Stelmach, B., Niesel, U., and Weiler, E. W. (1999). Structure-activity analyses reveal the existence of two separate groups of active octadecanoids in elicitation of the tendril-coiling response of Bryonia dioica Jacq. Planta 207, 470-479.
Böttcher, C., and Pollmann, S. (2009). Plant oxylipins: plant responses to 12-oxo-phytodienoic acid are governed by its specific structural and functional properties. FEBS J. 276, 4693-4704.

Böttcher, C., and Weiler, E. W. (2007). cyclo-Oxylipingalactolipids in plants: occurrence and dynamics. Planta 226, 629-637.

Browse, J. (2009). Jasmonate passes muster: a receptor and targets for the defense hormone. Annu. Rev. Plant Biol. 60, 183-205. 
Buseman, C,M., Tamura, P., Sparks, A. A., Baughman, E. J., Maatta, S., Zhao, J., Roth, M. R., Esch, S. W., Shah, J., Williams, T. D., and Welti, R. (2006). Wounding stimulates the accumulation of glycerolipids containing oxophytodienoic acid and dinor-oxophytodienoic acid in Arabidopsis leaves. Plant Physiol. 142, 28-39.

Chehab, E. W., Kaspi, R., Savchenko, T., Rowe, H., Negre-Zakharov, F., Kliebenstein, D., and Dehesh, K. (2008). Distinct roles of jasmonates and aldehydes in plant-defense responses. PLoS ONE 3, e1904. doi:10.1371/journal.pone.0001904

Chehab, E. W., Kim, S., Savchenko, T., Kliebenstein, D., Dehesh, K., and Braam, J. (2011). Intronic T-DNA insertion renders Arabidopsis opr3 a conditional jasmonic-acid producing mutant. Plant Physiol. 156, 770-778.

Chini, A., Fonseca, S., Fernandez, G., Adie, B., Chico, J. M., Lorenzo, O., Garcia-Casado, G., Lopez-Vidriero, I., Lozano, F. M., Ponce, M. R., Micol, J. L., and Solano, R. (2007). The JAZ family of repressors is the missing link in jasmonate signalling. Nature 448, 666-671.

Cruz Castillo, M., Martinez, C., Buchala, A., Metraux, J. P., and Leon, J. (2004). Gene-specific involvement of $\beta$-Oxidation in wound-activated responses in Arabidopsis. Plant Physiol. 135, 85-94.

Dave, A., Hernández, M. L., He, Z., Andriotis, V. M., Vaistij, F. E., Larson, T. R., and Graham, I. A. (2011). 12-Oxo-phytodienoic acid accumulation during seed development represses seed germination in Arabidopsis. Plant Cell 23, 583-599.

Duan, H., Huang, M. Y., Palacio, K., and Schuler, M. A. (2005). Variations in CYP74B2 (Hydroperoxide lyase) gene expression differentially affect hexenal signaling in the Columbia and Landsberg erecta ecotypes of Arabidopsis. Plant Physiol. 139, 1529-1544.

Ellinger, D., Stingl, N., Kubigsteltig, I. I., Bals, T., Juenger, M., Pollmann, S., Berger, S., Schuenemann, D., and Mueller, M. J. (2010). DONGLE and DEFECTIVE IN ANTHER DEHISCENCE lipases are not essential for wound- and pathogen-induced jasmonate biosynthesis: redundant lipases contribute to jasmonate production. Plant Physiol. 153, 114-127.

Eschen-Lippold, L., Altmann, S., Gebhardt, C., Göbel, C., Feussner, I., and Rosahl, S. (2010). Oxylipins are not required for $\mathrm{R}$ gene-mediated resistance in potato. Eur. J. Plant Pathol. 127, 437-442.

Farmer, E. E., and Davoine, C. (2007). Reactive electrophile species. Curr. Opin. Plant Biol. 10, 380-386.

Feys, B., Benedetti, C. E., Penfold, C. N., and Turner, J. G. (1994). Arabidopsis mutants selected for resistance to the phytotoxin coronatine are male sterile, insensitive to methyl jasmonate and resistant to a bacterial pathogen. Plant Cell 6, 751-759.

Footitt, S., Marquez, J., Schmuths, H., Baker, A., Theodoulou, F. L., and Holdsworth, M. (2006). Analysis of the role of COMATOSE and peroxisomal beta-oxidation in the determination of germination potential in Arabidopsis. J. Exp. Bot. 57, 2805-2814.

Heitz, T., Widemann, E, Lugan, R., Miesch, L., Ullmann, P., Désaubry, L., Holder, E., Grausem, B., Kandel, S., Miesch, M., Werck-Reichhart, D., and Pinot, F. (2012). Cytochromes P450 CYP94C1 and CYP94B3 catalyze two successive oxidation steps of the plant hormone jasmonoylisoleucine for catabolic turnover. $J$. Biol. Chem. 287, 6296-6306.

Hisamatsu, Y., Goto, N., Hasegawa, K., and Shigemori, H. (2003). Arabidopsides A and B, two new oxylipins from Arabidopsis thaliana. Tetrahedron Lett. 44, 5553-5556.

Hisamatsu, Y., Goto, N., Hasegawa, K., and Shigemori, H. (2006). Senescence-promoting effect of arabidopside A. Z. Naturforsch. CBiosci. 61, 363-366.

Hisamatsu, Y., Goto, N., Sekiguchi, M., Hasegawa, K., and Shigemori, H. (2005). Oxylipins arabidopsides C and D from Arabidopsis thaliana. J. Nat. Prod. 68, 600-603.

Hyun, Y., Choi, S., Hwang, H. J., Yu, J., Nam, S. J., Ko, J., Park, J. Y., Seo, Y. S., Kim, E. Y., Ryu, S. B., Kim, W. T., Lee, Y. H., Kang, H., and Lee, I. (2008). Cooperation and functional diversification of two closely related galactolipase genes for jasmonate biosynthesis. Dev. Cell 14, 183-192.

Ibrahim, A., Schütz, A. L., Galano, J. M., Herrfurth, C., Feussner, K., Durand, T., Brodhun, F., and Feussner, I. (2011). The alphabet of galactolipids in Arabidopsis thaliana. Front. Plant Sci. 2:95. doi:10.3389/fpls.2011.00095.

Imbusch, R., and Mueller, M. J. (2000). Analysis of oxidative stress and wound-inducible dinor isoprostanes $\mathrm{F}(1)$ (phytoprostanes $\mathrm{F}(1)$ ) in plants. Plant Physiol. 124, 1293-1304.

Ishiguro, S., Kawai-Oda, A., Ueda, J., Nishida, I., and Okada, K. (2001).
The DEFECTIVE IN ANTHER DEHISCENCE1 gene encodes a novel phospholipase A1 catalyzing the initial step of jasmonic acid biosynthesis, which synchronizes pollen maturation, anther dehiscence, and flower opening in Arabidopsis. Plant Cell 13, 2191-2209.

Itoh, A., and Howe, G. A. (2001). Molecular cloning of a divinyl ether synthase. Identification as a CYP74 cytochrome P-450. J. Biol. Chem. 276, 3620-3627.

Kanai, M., Nishimura, M., and Hayashi, M. (2010). A peroxisomal ABC transporter promotes seed germination by inducing pectin degradation under the control of ABI5. Plant J. 62, 936-947.

Kienow, L., Schneider, K., Bartsch, M., Stuible, H. P., Weng, H., Miersch, O., Wasternack, C., and Kombrink, E. (2008). Jasmonates meet fatty acids: functional analysis of a new acylcoenzyme A synthetase family from Arabidopsis thaliana. J. Exp. Bot. 59, 403-419.

Kitaoka, N., Matsubara, T., Sato, M. Takahashi, K., Wakuta, S., Kawaide, H., Matsui, H., Nabeta, K., and Matsuura, H. (2011). Arabidopsis CYP94B3 encodes jasmonyl-Lisoleucine 12-hydroxylase, a key enzyme in the oxidative catabolism of jasmonate. Plant Cell Physiol. 52, 1757-1765.

Koo, A. J. K., Chung, H. S., Kobayashi, Y. and Howe, G. A. (2006). Identification of a peroxisomal acyl-activating enzyme involved in the biosynthesis of jasmonic acid in Arabidopsis. J. Biol. Chem. 281, 33511-33520.

Koo, A. J., Cooke, T. F., and Howe, G. A. (2011). Cytochrome P450 CYP94B3 mediates catabolism and inactivation of the plant hormone jasmonoyl-L-isoleucine. Proc. Natl. Acad. Sci. U.S.A. 108, 9298-9303.

Kourtchenko, O., Andersson, M. X., Hamberg, M., Brunnström, A., Göbel, C., McPhail, K. L., Gerwick, W. H., Feussner, I., and Ellerström, M. (2007). Oxo-phytodienoic acid-containing galactolipids in Arabidopsis: jasmonate signaling dependence. Plant Physiol. 145, 1658-1669.

Li, L., Zhao, Y., McCaig, B. C., Wingerd, B. A., Wang, J., Whalon, M. E., Pichersky, E., and Howe, G. A. (2004). The tomato homolog of CORONATINE-INSENSITIVE1 is required for the maternal control of seed maturation, jasmonatesignaled defense responses, and glandular trichome development. Plant Cell 16, 126-143.
Mosblech, A., Feussner, I., and Heilmann, I. (2009). Oxylipins: structurally diverse metabolites from fatty acid oxidation. Plant Physiol. Biochem. 47, 511-517.

Mueller, S., Hilbert, B., Dueckershoff, K., Roitsch, T., Krischke, M., Mueller M. J., and Berger, S. (2008). General detoxification and stress responses are mediated by oxidized lipids through TGA transcription factors in Arabidopsis. Plant Cell 20 768-785.

Penfield, S., Li, Y., Gilday, A. D., Graham, S., and Graham, I. A. (2006). Arabidopsis ABA INSENSITIVE4 regulates lipid mobilization in the embryo and reveals repression of seed germination by the endosperm. Plant Cell 18, 1887-1899.

Pinfield-Wells, H., Rylott, E. L., Gilday, A. D., Graham, S., Job, K., Larson, T. R., and Graham, I. A. (2005). Sucrose rescues seedling establishment but not germination of Arabidopsis mutants disrupted in peroxisomal fatty acid catabolism. Plant J. 43,861-872.

Pracharoenwattana, I., Cornah, J. E. and Smith, S. M. (2005). Arabidopsis peroxisomal citrate synthase is required for fatty acid respiration and seed germination. Plant Cell 17, 2037-2048.

Ribot, C., Zimmerli, C., Farmer, E. E., Reymond, P., and Poirier, Y. (2008). Induction of the Arabidopsis PHO1;H10 gene by 12-oxo-phytodienoic acid but not jasmonic acid via a CORONATINE INSENSITIVE1-dependent pathway. Plant Physiol. 147, 696-706.

Sanders, P. M., Lee, P. Y., Biesgen, C., Boone, J. D., Beals, T. P., Weiler, E. W., and Goldberg, R. B. (2000). The Arabidopsis DELAYED DEHISCENCE1 gene encodes an enzyme in the jasmonic acid synthesis pathway. Plant Cell 12, 1041-1061.

Schäfer, M., Fischer, C., Baldwin, I. T., and Meldau, S. (2011). Grasshopper oral secretions increase salicylic acid and abscic acid levels in wounded leaves of Arabidopsis thaliana. Plant Signal. Behav. 6, 1256-1258.

Schaller, F., Biesgen, C., Müssig, C., Altmann, T., and Weiler, E. W. (2000). 12-Oxophytodienoate reductase 3 (OPR3) is the isoenzyme involved in jasmonate biosynthesis. Planta 210, 979-984.

Schilmiller, A. L., Koo, A. J., and Howe, G. A. (2007). Functional diversification of acyl-coenzyme A oxidases in jasmonic acid biosynthesis and action. Plant Physiol. 143, 812-824. 
Schneider, K., Kienow, L., Schmelzer, E., Colby, T., Bartsch, M., Miersch, O., Wasternack, C., Kombrink, E., and Stuible, H. P. (2005). A new type of peroxisomal acyl-Coenzyme A synthetase from Arabidopsis thaliana has the catalytic capacity to activate biosynthetic precursors of jasmonic acid. J. Biol. Chem. 280, 13962-13972.

Staswick, P. E., Su, W., and Howell, S. H. (1992). Methyl jasmonate inhibition of root growth and induction of a leaf protein are decreased in an Arabidopsis thaliana mutant. Proc. Natl. Acad. Sci. U.S.A. 89, 6837-6840.

Staswick, P. E., and Tiryaki, I. (2004). The oxylipin signal jasmonic acid is activated by an enzyme that conjugates it to isoleucine in Arabidopsis. Plant Cell 16, 2117-2127.

Stelmach, B. A., Müller, A., Hennig, P., Gebhardt, S., Schubert-Zsilavecz, M., and Weiler, E. W. (2001). A novel class of oxylipins, sn1-O(12-Oxophytodienoyl)-sn2-O(hexadecatrienoyl)-monogalactosyl diglyceride, from Arabidopsis thaliana. J. Biol. Chem. 276, 12832-12838.

Stintzi, A., and Browse, J. (2000). The Arabidopsis male-sterile mutant, opr3, lacks the 12-oxophytodienoic acid reductase required for jasmonate synthesis. Proc. Natl. Acad. Sci. U.S.A. 97, 10625-10630.

Stintzi, A., Weber, H., Reymond, P., Browse, J., and Farmer, E. E. (2001). Plant defense in the absence of jasmonic acid: the role of cyclopentanones. Proc. Natl. Acad. Sci. U.S.A. 98, 12837-12842.

Taki, N., Sasaki-Sekimoto, Y., Obayashi, T., Kikuta, A., Kobayashi, K., Ainai, T., Yagi, K., Sakurai, N., Suzuki, H., Masuda, T., Takamiya, K., Shibata, D., Kobayashi, Y., and Ohta, H. (2005). 12-Oxo-Phytodienoic acid triggers expression of a distinct set of genes and plays a role in wound-induced gene expression in Arabidopsis. Plant Physiol. 139, 1268-1283.

Theodoulou, F. L., Job, K., Slocombe, S. P., Footitt, S., Holdsworth, M., Baker, A., Larson, T. R., and Graham, I. A. (2005). Jasmonic acid levels are reduced in COMATOSE ATP-binding cassette transporter mutants. Implications for transport of jasmonate precursors into peroxisomes. Plant Physiol. 137, 835-840.

Thines, B., Katsir, L., Melotto, M., Niu, Y., Mandaokar, A., Liu, G., Nomura, K., He, S. Y., Howe, G. A., and Browse, J. (2007). JAZ repressor proteins are targets of the SCFCOIl complex during jasmonate signalling. Nature 448, 661-665.

Vick, B. A., and Zimmerman, D. C. (1983). The biosynthesis of jasmonic acid: a physiological role for plant lipoxygenase. Biochem. Biophys. Res. Commun. 111, 470-477.

$\mathrm{Vu}$, H. S., Tamura, P., Galeva, N. A., Chaturvedi, R., Roth, M. R., Williams, T. D., Wang, X., Shah,
J., and Welti, R. (2012). Direct infusion mass spectrometry of oxylipin-containing Arabidopsis membrane lipids reveals varied patterns in different stress responses. Plant Physiol. 158, 324-339.

Wasternack, C. (2007). Jasmonates: an update on biosynthesis, signal transduction and action in plant stress response, growth and development. Ann. Bot. 100, 681-697.

Wasternack, C., and Kombrink, E. (2010). Jasmonates: structural requirements for lipid-derived signals active in plant stress responses and development. ACS Chem. Biol. 5, 63-77.

Weber, H., Chételat, A., Caldelari, D. and Farmer, E. E. (1999). Divinyl ether fatty acid synthesis in late blight-diseased potato leaves. Plant Cell 11, 485-494.

Weber, H., Chételat, A., Reymond, P. and Farmer, E. E. (2004). Selective and powerful stress gene expression in Arabidopsis in response to malondialdehyde. Plant J. 37, 877-888.

Weber, H., Vick, B. A., and Farmer, E. E. (1997). Dinor-oxo-phytodienoic acid: a new hexadecanoid signal in the jasmonate family. Proc. Natl. Acad. Sci. U.S.A. 94, 10473-10478.

Weiler, E. W., Albrecht, T., Groth, B., Xia, Z.-Q., Luxem, M., Liss, H., Andert, L., and Spengler, P. (1993). Evidence for the involvement of jasmonates and their octadecanoid precursors in the tendril coiling response of Bryonia dioica. Phytochemistry 32, 591-600.
Xie, D. X., Feys, B. F., James, S., Nieto-Rostro, M., and Turner, J. G. (1998). COI1: an Arabidopsis gene required for jasmonate-regulated defense and fertility. Science 280, 1091-1094.

Yan, Y., Stolz, S., Chételat, A., Reymond, P., Pagni, M., Dubugnon, L., and Farmer, E. E. (2007). A downstream mediator in the growth repression limb of the jasmonate pathway. Plant Cell 19, 2470-2483.

Conflict of Interest Statement: The authors declare that the research was conducted in the absence of any commercial or financial relationships that could be construed as a potential conflict of interest.

Received: 20 December 2011; accepted: 19 February 2012; published online: 08 March 2012.

Citation: Dave $A$ and Graham IA (2012) Oxylipin signaling: a distinct role for the jasmonic acid precursor cis-(+)-12-oxo-phytodienoic acid (cisOPDA). Front. Plant Sci. 3:42. doi 10.3389/fpls.2012.00042

This article was submitted to Frontiers in Plant Physiology, a specialty of Frontiers in Plant Science.

Copyright (c) 2012 Dave and Graham. This is an open-access article distributed under the terms of the Creative Commons Attribution Non Commercial License, which permits non-commercial use, distribution, and reproduction in other forums, provided the original authors and source are credited. 